Entzündungsparameter

\section{Einfluss von Metformin und/oder Insulin auf hsCRP}

Hintergrund: Bei Diabetes mellitus finden sich oft erhöhte Entzündungsparameter. Ob diese durch unterschiedliche Therapien zu beeinflussen sind, wurde nun untersucht.

Methoden: In der randomisierten USamerikanischen Studie mit $2 \times 2$ faktoriellem Design erhielten insgesamt 500 Typ-2-Diabetiker mit nicht optimaler Stoffwechsellage (durchschnittlich 2 Jahre Diabetesdauer) und erhöhten hsCRP-

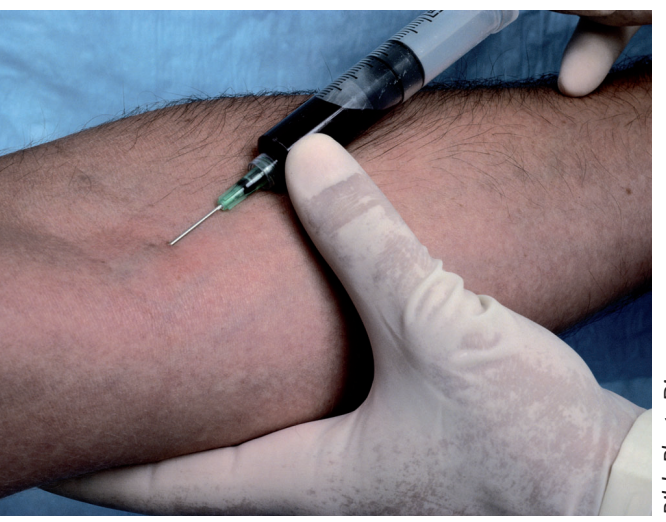

Spiegeln entweder open-label Insulin Glargin oder Placebo-kontrolliert Metformin. Nach Rekrutierung von Oktober 2006 bis Dezember 2008 erfolgte die Randomisierung in eine der 4 Studiengruppen (nur Placebo, Placebo und Insulin Glargin; nur Metformin, Metformin und Insulin Glargin) mit Dosistitrierung auf Nüchtern-Blutglukosewerte unter $110 \mathrm{mg} / \mathrm{dl}$. Primärer Endpunkt war die Änderung der hsCRP-Spiegel, sekundäre Endpunkte die Änderungen der Spiegel von Interleukin-6 (IL-6) sowie löslichem Tumor-Nekrose-Faktor-Rezeptor-2 (sTNFr2) während der Studiendauer von 14 Wochen.

Ergebnisse: Die Blutglukose- und $\mathrm{HbA}_{1 \mathrm{c}}{ }^{-}$ Werte sanken signifikant unter Verum im Vergleich zu Placebo (jeweils $\mathrm{p}<0,001)$. Bei der Reduktion der hsCRPSpiegel wurden keine Unterschiede zwischen den Insulin behandelten $(-11,8 \%$; $95 \%-\mathrm{KI}-18,7$ bis $-4,4 \%)$ und den nicht Insulin behandelten Patienten (-17,5\%; $95 \%-\mathrm{KI}-23,9$ bis $-10,5 \%)$ so- wie zwischen den Metformin (-18,1\%; $95 \%-\mathrm{KI}-24,4$ bis $-11,1 \%$ ) und den nicht mit Metformin behandelten Patienten $(-11,2 \%$; $95 \%-\mathrm{KI}-18,1$ bis $-3,7 \%)$ beobachtet. Bei separater Betrachtung der 4 Behandlungsgruppen betrug die Reduktion der hsCRP-Spiegel unter Metformin -16,1\% (95\%-KI -25,1 bis $-6,1 \%$ ), unter Metformin und Insulin $-20,1 \%$ (95\%-KI $-28,8$ bis $-10,4 \%)$, unter nur Placebo (-19,0\%; 95\%-KI -27,8 bis $-9,1 \%)$. Im Vergleich dazu sanken die hsCRP-Spiegel in der nur mit Insulin behandelten Gruppe um lediglich $-2,9 \%$ (95\%-KI -13,2 bis 8,6\%). Bei Analyse der IL-6- and sTNFr2-Spiegel waren die Ergebnisse vergleichbar.

Folgerung: Bei Typ-2-Diabetikern mit unbefriedigender Stoffwechsellage kurz nach Manifestation lassen sich weder durch Metformin, noch durch Insulin oder durch die Kombination von beidem die Spiegel von hsCRP, IL-6 und sTNFr2 im Vergleich zu Placebo signifikant beeinflussen.

Dr. Winfried Keuthage, Münster

Quelle: Pradhan AD, Everett BM, Cook NR et al. Effects of Initiating Insulin and Metformin on Glycemic Control and Inflammatory Biomarkers Among Patients With Type 2 Diabetes. JAMA 2009; 302: 1186-1194 